\title{
The Coefficient of Variation of Red Blood Cell Distribution Width Combined with Cancer Antigen 125 Predicts Postoperative Overall Survival in Endometrial Cancer
}

\author{
Wenhui Zhong ${ }^{1} *$ \\ Chunyu Zhou ${ }^{2, *}$ \\ Lufei Chen' \\ Zhenna Wang ${ }^{3}$ \\ Hongxing Lin' \\ Kunhai Wu' \\ Sujiao Zhang ${ }^{4}$
}

'Clinical Laboratory, Fujian Maternity and Child Health Hospital, Affiliated Hospital of Fujian Medical University, Fuzhou, People's Republic of China; ${ }^{2}$ Nursing Department, Fujian Medical University Union Hospital, Fuzhou, People's Republic of China; ${ }^{3}$ Department of Gynecology, Fujian Maternity and Child Health Hospital, Affiliated Hospital of Fujian Medical University, Fuzhou, People's Republic of China; ${ }^{4}$ Department of Pathology, Fujian Maternity and Child Health Hospital, Affiliated Hospital of Fujian Medical University, Fuzhou,

People's Republic of China

*These authors contributed equally to this work
Correspondence: Wenhui Zhong

Clinical Laboratory, Fujian Maternity and Child Health Hospital, Affiliated Hospital of Fujian Medical University, No. 18 Daoshan Road, Fuzhou, 35000I, Fujian,

People's Republic of China

Email289492316@qq.com
Purpose: This study assessed the prognostic value of red blood cell distribution width (RDW) and cancer antigen 125 (CA125) in predicting the prognosis of endometrial cancer (EC) patients.

Patients and Methods: In this study, we included 525 patients with EC between January 2013 and January 2019. Demographic and clinical indicators were collected, and the receiver operating characteristics curve (ROC) and cutoff values were calculated between the early and advanced stages of EC. Independent risk factors associated with EC prognosis were assessed using Cox regression analyses and the Kaplan-Meier method.

Results: Compared to women in the early stage of EC, women with advanced stage had significantly elevated RDW coefficient of variation (RDW-CV) and CA125 levels and lower mean corpuscular volume (MCV) and mean corpuscular hemoglobin $(\mathrm{MCH})$ (both $\mathrm{P}<0.05)$. Consequently, RDW-CV and CA125 were found to be independent risk factors for EC by using ROC curve and multivariate logistic regression analysis. The survival analysis curve was used to assess the diagnostic value of RDW-CV, CA125, and their combination in the prognosis of EC. The results showed that patients with high expression of RDW-CV and CA125 had worse overall survival than those with low expression. Moreover, multivariate Cox regression analysis indicated that $\mathrm{RDW}-\mathrm{CV}+\mathrm{CA} 125=2$ was an independent prognostic factor.

Conclusion: These findings suggest that CA125 combined with RDW-CV has a good prognostic value for $\mathrm{EC}$. Thus, the $\mathrm{RDW}-\mathrm{CV}+\mathrm{CA} 125$ score is a promising prognostic marker for the clinical decision-making process regarding EC outcomes.

Keywords: CA125, RDW-CV, endometrial cancer, prognosis

\section{Introduction}

Endometrial cancer (EC) is the most common gynecological malignancy in developed countries, and its incidence is increasing each year. ${ }^{1}$ Patients with early stage EC have a good prognosis, with a 5-year survival rate of 74-91\%, while patients diagnosed with stage III or IV EC have a 5-year survival rate of 57-66\% and 20$26 \%$, respectively. ${ }^{2}$ According to statistics, EC has a long onset period and high insidiousness, meaning its symptoms are not meaningful at the early stages of the disease. $^{3}$ Moreover, studies have shown that $10-20 \%$ of patients with early EC and $50-70 \%$ of patients with advanced EC have a poor prognosis. ${ }^{4}$ Therefore, 
identification of reliable and feasible biomarkers is needed for the detection of EC patients with poor prognosis, development of individualized treatments, and implementation of follow-up protocols. At present, cancer antigen 125 (CA125), is associated with the prognosis of EC. ${ }^{5}$ However, when patients with non-EC suffer from endometriosis, ${ }^{6}$ hepatitis, ${ }^{7}$ or when the body is in the inflammatory response period, the level of serum CA125 will also increase to varying degrees. Therefore, the specificity of CA125 as a tumor marker for EC is not high, and it is not recommended to use CA125 alone as a prognostic indicator of EC.

Red blood cell (RBC) distribution width (RDW) is a parameter reflecting the variation degree of red blood cell volume, which is often used in the clinical diagnosis of anemia types such as iron deficiency anemia and thalassemia. ${ }^{8} \mathrm{He}$ et $\mathrm{al}^{9}$ confirmed that RDW was positively correlated with erythrocyte sedimentation rate (ESR) and C-reactive protein (CRP), suggesting that changes in RDW may be related to the inflammatory state of the body. Clinical data have also shown that chronic inflammation is closely related to the progression of cancer. ${ }^{10}$ Recent studies have reported the expression of RDW in various common tumors, such as breast cancer, ${ }^{11}$ ovarian cancer $^{12}$ and gastric cancer, ${ }^{13}$ and its role in the diagnosis or prognosis of such tumors. Other studies have shown that RBC count and anemia are associated with the prognosis of EC. ${ }^{14}$ However, the role of RDW in EC is not yet fully understood. Therefore, the present study aimed to investigate the role of RDW and CA125 (alone or in combination) in the prognosis of EC.

\section{Patients and Methods}

\section{Study Population}

This descriptive study is a retrospective study conducted in a single center. Data of all women who had undergone primary hysterectomy for EC were retrospectively collected from the Fujian Provincial Maternity and Children's Hospital between January 2013 and January 2019.

The exclusion criteria were as follows: (1) secondary malignancies $(n=4)$; (2) blood transfusion history within 3 months or complications with hematologic disease $(n=0)$; (3) radiotherapy or chemotherapy before surgery $(n=0)$; (4) history of hormonal therapy within 12 months $(n=0)$; (5) complications with all kinds of infectious diseases $(\mathrm{n}=0)$; (6) missing data for the variables or covariates analyzed in the present study $(n=15)$; (7) lost before follow-up $(n=6)$. Therefore, the eligible study cohort comprised 525 patients. Informed consent was obtained from the patients before treatment, and data collection was performed in accordance with the institutional guidelines. The protocol was approved by the Ethics Committee of the Fujian Provincial Maternity and Children's Hospital.

\section{Sample Size}

Our study included 525 patients with EC, from which clinical data and immunohistochemical pathological data were collected. We performed a sample size calculation according to two independent design data calculation formulas post hoc. The calculated sample size was sufficient, and the power was 0.8 .

\section{Data Collection}

Demographic and clinical data were collected from the Fujian Provincial Maternity and Children's Hospital information system, and included age at surgery, body mass index (BMI), RDW coefficient of variation (RDW$\mathrm{CV}$ ) and standard deviation (RDW-SD), Mean Corpuscular Volume (MCV), Mean Corpuscular Hemoglobin (MCH), Mean Corpuscular Hemoglobin Concerntration (MCHC), white blood cells (WBC), neutrophilic granulocyte (NE), RBC, hemoglobin ( $\mathrm{Hb}$ ), CA125, cancer antigen 199 (CA199) and 153 (CA153), Carcinoma Embryonic Antigen (CEA), Alpha Fetal Protein (AFP), menopause, history of diabetes, hypertension, and International Federation of Gynecology and Obstetrics (FIGO) stage.

\section{Follow-Up}

We followed up all included EC patients for survival outcomes. The last follow-up time was on January, 2020. The median follow-up period was 45.6 months (range 2.4-88.8 months). Overall survival (OS) was defined as the time from surgery to the date of death or to the last follow-up.

\section{Statistical Analysis}

Data distribution was assessed using the KolmogorovSmirnov test. Continuous variables are presented as means $\pm \mathrm{SD}$ or as median (interquartile range), as appropriate, depending on whether the data were normally distributed; dichotomous variables are expressed as percentages. Continuous variables were compared using analyses of variance, Kruskal-Wallis tests, unpaired 
$t$-tests, or Mann-Whitney $U$-tests, as appropriate. Categorical variables were compared using chi-squared or Fisher's exact tests, as appropriate. To identify the factors associated with EC, receiver operating characteristic (ROC) curves were generated for each continuous variable. The areas under the curve (AUCs) are provided with their sensitivity, specificity, and 95\% confidence intervals (CIs). Cox regression analysis and KaplanMeier analysis were used for histological and clinical factors. Adjusted risk estimates were obtained using logistic regression models and accounted for the variables used for matching. Significance was set at $P \leq 0.05$. All analyses were performed using SPSS software (version 22.0; IBM Corp., Armonk, NY, USA).

\section{Results}

\section{Patient Characteristics and Independent Prognostic Factors}

Our study included 525 patients with EC and collected relevant clinical data for analysis. As the prognosis of patients with early- and advanced-stage EC is different, we divided the included EC patients into two groups (I-II vs III-IV) for analysis according to the FIGO stage. In general, there were no differences between the two groups with respect to age, $\mathrm{BMI}, \mathrm{WBC}, \mathrm{NE}, \mathrm{RBC}, \mathrm{HB}, \mathrm{MCHC}$, RDW-SD, AFP, CEA, menopause, history of diabetes, and hypertension (all $\mathrm{P}>0.05$; Table 1). However, compared to women in stages I-II, women with advanced stage EC had significantly elevated RDW-CV, CA125, CA199, CA153, and lower $\mathrm{MCV}$ and $\mathrm{MCH}$ (both $\mathrm{P}<0.05$, Table 1). To explore the independent high-risk factors of $\mathrm{EC}$, the significantly different factors were evaluated by ROC curve analysis. The cutoff values for statistically significant EC indicators were identified (Figure 1). Based on these cutoff values, RDW-CV and CA125 were found to be independent risk factors for EC using univariate and multivariate binary logistic regression analyses ( $\mathrm{P}$ $<0.05$, Table 2).

\section{Correlations Between Clinicopathological Features and RDW-CV+CAI 25}

Higher CA125 and RDW-CV levels were independent risk factors for the progression of EC stages; as so, based on CA125 and RDW-CV levels, we divided EC patients into the following three subgroups: both low CA125 and RDW-CV (assigned a score of 0), either low CA125 or low RDW-CV (assigned a score of 1), and both high
CA125 and RDW-CV (assigned a score of 2). Table 3 shows the relationship between RDW-CV+CA125 and some clinical indicators. The results showed that higher RDW-CV+CA125 levels were positively associated with the FIGO stage $(\mathrm{P}<0.001$, Table 3$)$. There were no differences among the three subgroups with respect to age, BMI, menopause, history of diabetes, and hypertension. (all $\mathrm{P}>0.05$, Table 3).

\section{Influence of the RDW-CV+CAI 25 on the OS of EC}

Univariate Cox regression analysis showed that RDW-CV + CA125 and FIGO stages were associated with overall survival (OS) of EC (all $\mathrm{P}<0.05$, Table 4). Multivariate Cox regression analysis indicated that RDW-CV+CA125 $=2$ was an independent prognostic factor (HR, 5.488; 95\% $\mathrm{CI}=1.081-27.855 ; \mathrm{P}=0.040$, Table 4). The KaplanMeier curve showed that the 3-year OS rates of EC was 96.4\% (95\% CI, 95.6-97.2) and the 5-year OS rates was 95.1\% (95\% CI, 93.9-96.3). In addition, the results showed that the high CA125 and RDW-CV subgroup $(\mathrm{RDW}-\mathrm{CV}+\mathrm{CA} 125=2)$ had the lowest OS $(\mathrm{P}<0.05$, Figure 2). Moreover, when stratified by FIGO stage, there were also significant differences in OS with RDW$\mathrm{CV}+\mathrm{CA} 125$ of 0,1 and 2 in the Stage I-II and III-IV subgroups (all $\mathrm{P}<0.05$, Figure 2).

\section{Discussion}

The blood parameters of patients with EC at different FIGO stages were investigated. Significant differences in RDW-CV and CA125 levels were observed between the early and advanced stages of EC. Moreover, multivariate Cox regression analysis indicated that high CA125 and RDW-CV (RDW-CV+CA125 = 2) were independent prognostic factors. No similar findings have been reported in the literature.

The RDW is a common parameter in clinical hematology, and RDW-CV and RDW-SD can describe the heterogeneity of erythrocyte volume. $\mathrm{RDW}-\mathrm{CV}$ is obtained from the relative ratio of the RDW-SD to the mean $\mathrm{RBC}$ volume, while RDW-SD is $20 \%$ above the baseline RBC volume distribution curve width. Although the exact mechanism of the association between RDW and malignant tumors has not yet been clarified, most studies have shown that the mechanism of RDW on tumors is mainly manifested by inflammation, ${ }^{15}$ malnutrition ${ }^{16}$ and oxidative stress. ${ }^{17}$ First, malignancy is usually accompanied by systemic 
Table I The Characteristics of Clinical Indicators in EC with Early and Advanced Stage

\begin{tabular}{|c|c|c|c|}
\hline Parameter & $I-I I(n=446)$ & III-IV(n=5I) & $\mathbf{P}$ \\
\hline Age at surgery, median (Range), years & $54(49-59)$ & $53(49-6 I)$ & 0.789 \\
\hline BMI, median (Range), kg/m2 & $24.12(22.21-26.56)$ & $24.45(22.36-26.16)$ & 0.795 \\
\hline WBC, median (Range), $10^{9} / \mathrm{L}$ & $6.13(5.01-7.16)$ & $6.21(5.28-7.4 I)$ & 0.286 \\
\hline $\mathrm{NE}$, median (Range), $10^{9} / \mathrm{L}$ & $3.38(2.7 I-4.33)$ & $3.7 \mathrm{I}(2.84-4.92)$ & 0.130 \\
\hline RBC, median (Range), $10^{9} / \mathrm{L}$ & $4.27(4.00-4.60)$ & $4.16(3.94-4.51)$ & 0.210 \\
\hline HB, median (Range), g/L & $126.00(\mid 16.25-134.00)$ & $121.50(111.00-133.25)$ & 0.130 \\
\hline MCV, median (Range), fL & $87.6(83.3-90.4)$ & $85.0(81.5-89.4)$ & 0.034 \\
\hline $\mathrm{MCH}$, median (Range), pg & $29.6(28.0-30.8)$ & $28.7(26.8-29.9)$ & 0.010 \\
\hline $\mathrm{MCHC}$, median (Range), g/L & $338(329-344)$ & $334(327-342)$ & 0.167 \\
\hline RDW-CV, median (Range), \% & $12.5(12.1-13.2)$ & $13.2(\mid 2.65-15.55)$ & 0.001 \\
\hline RDW-SD, median (Range), fL & $40.3(38.8-42.5)$ & $41.2(38.93-43.68)$ & 0.185 \\
\hline CAI25, median (Range), U/mL & $18(11.60-28.58)$ & $38.85(19.05-122.95)$ & $<0.001$ \\
\hline CA199, median (Range), U/mL & $12.96(6.99-28.63)$ & $23.63(11.52-68.84)$ & $<0.001$ \\
\hline CAI53, median (Range), U/mL & $8.8(6.7-12.7)$ & |3.25(8.83-2|.58) & $<0.001$ \\
\hline AFP, median (Range), ng/mL & $2.46(1.80-3.36)$ & $2.27(1.77-3.28)$ & 0.467 \\
\hline CEA, median (Range), ug/L & $1.71(1.17-2.56)$ & $1.75(1.19-2.40)$ & 0.097 \\
\hline \multicolumn{4}{|l|}{ Menopause status, n(\%) } \\
\hline Premenopausal & 223 & 23 & 0.507 \\
\hline Postmenopausal & 223 & 28 & \\
\hline \multicolumn{4}{|l|}{ History of diabetes, $n(\%)$} \\
\hline Yes & 354 & 39 & 0.629 \\
\hline No & 92 & 12 & \\
\hline \multicolumn{4}{|l|}{ History of hypertension, $n(\%)$} \\
\hline Yes & 267 & 36 & 0.137 \\
\hline No & 169 & 15 & \\
\hline
\end{tabular}

Note: $\mathrm{P}<0.05$ suggests significantly different.

Abbreviations: BMI, body mass index; WBC, white blood cell; NE, neutrophil; RBC, red blood cell; HB, hemoglobin; MCV, mean corpuscular volume; MCH, mean corpuscular hemoglobin; MCHC, mean corpuscular hemoglobin concentration; RDW-CV, The coefficient of variation of red blood cell distribution; RDW-SD, standard deviation of red blood cell distribution; CAI25, cancer antigen 125; CAI99, cancer antigen 199; CAI53, cancer antigen I53; AFP, alpha fetal protein; CEA, A carcinoma embryonic antigen.

inflammation. In recent years, a number of studies have shown that there is a positive correlation between RDW and inflammatory markers. ${ }^{9,15}$ A possible mechanism is that inflammatory responses and inflammatory factors affect iron metabolism, shorten the life span of RBC, and lead to the release of a large number of immature RBC and entry of bone marrow cells into the surrounding blood circulation. ${ }^{15}$ In addition, inflammatory factors can lead to ineffective hematopoiesis in the bone marrow, resulting in different sizes of $\mathrm{RBC}$ in the peripheral blood, leading to increased RDW.
Chronic inflammation will keep patients in a state of longterm high level of oxidative stress, resulting in the decrease of RBC and the release of immature RBC into the blood, leading to an increase in RDW. ${ }^{17}$ In addition, patients with advanced cancer are often associated with emaciation and malnutrition, leading to deficiency of nutrients such as vitamin B12 or folic acid, which leads to anemia and an increase in RDW. ${ }^{16}$ In recent years, many studies have shown that $\mathrm{RDW}$ is associated with the prognosis of breast cancer, ${ }^{11}$ ovarian cancer ${ }^{12}$ and gastric cancer. ${ }^{13}$ Zhang et $\mathrm{al}^{18}$ showed 

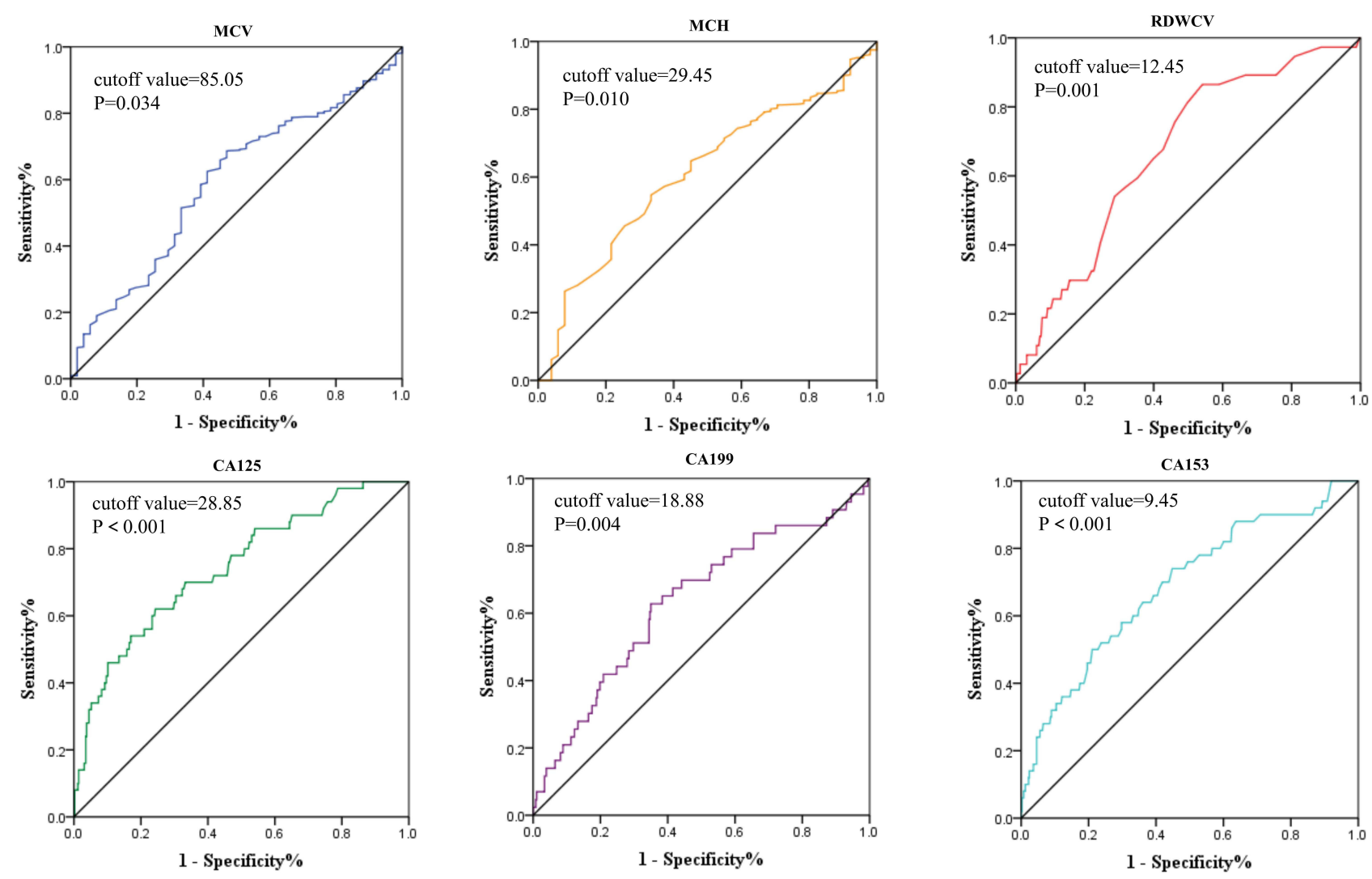

Figure I ROC curves of MCV, MCH, RDW-CV, CAI25, CA199, CAI53. P<0.05 suggests significantly different.

that RDW was an independent risk factor for EC and had focus only on RDW, without subdividing RDW into RDWa high diagnostic value. Few relevant studies have been CV and RDW-SD. Our study analyzed RDW-CV and RDWconducted on the RDW of EC, and most current studies SD in EC patients, and the results showed that RDW-CV was

Table 2 Univariate and Multivariate Logistic Regression Analyses for Stage

\begin{tabular}{|c|c|c|c|c|c|}
\hline \multicolumn{2}{|c|}{ Parameter } & \multirow{3}{*}{$\begin{array}{c}\text { Univariable } \\
\text { OR }(95 \% \mathrm{CI}) \\
0.406(0.226-0.769)\end{array}$} & \multirow{3}{*}{$\begin{array}{c}\mathbf{P}_{\mathbf{I}} \\
0.003\end{array}$} & \multirow{3}{*}{$\begin{array}{c}\text { Multivariable } \\
\text { OR }(95 \% \mathrm{Cl}) \\
0.609(0.192-1.938)\end{array}$} & \multirow{3}{*}{$\begin{array}{c}\mathbf{P}_{\mathbf{2}} \\
0.609\end{array}$} \\
\hline & & & & & \\
\hline MCV & $\begin{array}{l}\leq 85.05^{R} \\
>85.05\end{array}$ & & & & \\
\hline $\mathrm{MCH}$ & $\begin{array}{l}\leq 29.45^{R} \\
>29.45\end{array}$ & $0.4 \mid 4(0.225-0.764)$ & 0.005 & I. I74(0.364-3.788) & 0.789 \\
\hline RDW-CV & $\begin{array}{l}\leq 12.45^{R} \\
>12.45\end{array}$ & $5.42 \mid(2.059-\mid 4.277)$ & 0.001 & $4.635(I .437-\mid 4.95 I)$ & 0.010 \\
\hline $\mathrm{CA} / 25$ & $\begin{array}{l}\leq 28.85^{R} \\
>28.85\end{array}$ & $5.085(2.755-9.383)$ & $<0.001$ & $3.784(1.610-8.894)$ & 0.002 \\
\hline CA199 & $\begin{array}{l}\leq 18.88^{R} \\
>18.88\end{array}$ & $3.136(1.632-6.025)$ & 0.001 & $2.27 I(0.965-5.342)$ & 0.060 \\
\hline CAI53 & $\begin{array}{l}\leq 9.45^{R} \\
>9.45\end{array}$ & $3.508(1.81 \mathrm{I}-6.794)$ & $<0.001$ & $2.127(0.893-5.062)$ & 0.088 \\
\hline
\end{tabular}

Note: $\mathrm{P}<0.05$ suggests significantly different.

Abbreviations: $\mathrm{MCV}$, mean corpuscular volume; $\mathrm{MCH}$, mean corpuscular hemoglobin; RDW-CV, the coefficient of variation of red blood cell distribution; CAI25, cancer antigen 125; CAI99, cancer antigen 199; CAI53, cancer antigen 153; R, references group. 
Table 3 Associations of RDW+CAI25 with Clinical Characteristics

\begin{tabular}{|c|c|c|c|c|c|}
\hline \multicolumn{2}{|l|}{ Parameters } & \multicolumn{3}{|c|}{$\begin{array}{l}\text { RDWCV } \\
+ \text { CAI25, n }\end{array}$} & \multirow[t]{2}{*}{$\mathbf{P}$} \\
\hline & & \multirow{3}{*}{$\begin{array}{c}0 \\
60 \\
45\end{array}$} & \multirow{3}{*}{$\begin{array}{c}\text { I } \\
107 \\
67\end{array}$} & \multirow{3}{*}{$\begin{array}{c}\mathbf{2} \\
34 \\
25\end{array}$} & \\
\hline Age(years) & $\leq 55$ & & & & 0.735 \\
\hline & $>55$ & & & & \\
\hline \multirow[t]{2}{*}{ BMI $\left(\mathrm{kg} / \mathrm{m}^{2}\right)$} & $\leq 25$ & 55 & 106 & 35 & 0.326 \\
\hline & $>25$ & 50 & 67 & 23 & \\
\hline \multirow[t]{2}{*}{ FIGO stage } & I-II & 100 & 156 & 38 & $<0.001$ \\
\hline & III-IV & 1 & 16 & 19 & \\
\hline \multirow[t]{2}{*}{ Menopause status } & Premenopausal & 43 & 88 & 34 & 0.139 \\
\hline & Postmenopausal & 62 & 92 & 26 & \\
\hline \multirow[t]{2}{*}{ History of diabetes } & Yes & 20 & 41 & II & 0.657 \\
\hline & No & 85 & 139 & 49 & \\
\hline \multirow{2}{*}{$\begin{array}{l}\text { History of } \\
\text { hypertension }\end{array}$} & Yes & 42 & 72 & 20 & 0.629 \\
\hline & No & 63 & 108 & 40 & \\
\hline
\end{tabular}

Note: $P<0.05$ suggests significantly different.

Abbreviations: BMI, body mass index; FIGO, International Federation of Gynecology and Obstetrics; RDW-CV, the coefficient of variation of red blood cell distribution; CAI25, cancer antigen 125 .

associated with the stage of EC, with a higher level of RDW$\mathrm{CV}$ was a poor prognostic factor for EC. In contrast, there was no significant difference in RDW-SD levels between patients with early and advanced EC, and the mechanisms underlying this outcome need to be further investigated.

CA125 is a glycoprotein secreted by coelomic epithelial cells during embryonic development. It is widely found in endothelial cells and mesothelial cells of the female reproductive system. When these cells are stimulated by inflammation or damaged by tumor cells, the intercellular connections or basement membrane will be damaged, resulting in the release of CA125 into the blood and its high expression. Therefore, there may be varying degrees of elevation in the serum of healthy women or patients with tumor lesions. Currently, CA125 is closely related to the occurrence and development of EC and it is often used as a tumor marker for EC. ${ }^{19}$ Consistent with previous studies, our results suggest that CA125 is an adverse prognostic factor for EC. However, the specificity of CA125 as a tumor marker of EC is not high because the serum CA125 level will increase to varying degrees when nonEC patients suffer from endometriosis, ${ }^{6}$ hepatitis ${ }^{7}$ and tuberculosis, ${ }^{20}$ when pregnant, or when in the inflammatory response period. Therefore, in the present study, we combined RDW-CV with CA125 for prognosis analysis, and the results showed that compared with CA125 or RDW-CV alone, $\mathrm{RDW}-\mathrm{CV}+\mathrm{CA} 125$ had a better prognostic value.

Table 4 Univariate and Multivariate Cox Proportional Hazards Regression Analyses for OS

\begin{tabular}{|c|c|c|c|c|c|}
\hline \multicolumn{2}{|l|}{ Parameter } & $\begin{array}{c}\text { Univariable } \\
\text { HR }(95 \% \mathrm{Cl})\end{array}$ & $\mathbf{P}$ & $\begin{array}{l}\text { Multivariable } \\
\text { HR }(95 \% \mathrm{Cl})\end{array}$ & $\mathbf{P}$ \\
\hline MCV & $\begin{array}{l}\leq 85.05^{R} \\
>85.05\end{array}$ & $2.902(0.851-9.903)$ & 0.086 & - & - \\
\hline $\mathrm{MCH}$ & $\begin{array}{l}\leq 29.45^{\mathrm{R}} \\
>29.45\end{array}$ & $1.379(0.564-3.375)$ & $0.48 I$ & - & - \\
\hline CAI99 & $\begin{array}{l}\leq 18.88^{R} \\
>18.88\end{array}$ & $0.90 I(0.333-2.435)$ & 0.836 & - & - \\
\hline CAI53 & $\begin{array}{l}\leq 9.45^{R} \\
>9.45\end{array}$ & $1.194(0.485-2.937)$ & 0.700 & - & - \\
\hline RDWCV+CAI 25 & $\begin{array}{l}0^{R} \\
1 \\
2\end{array}$ & $\begin{array}{c}- \\
3.256(0.722-14.689) \\
6.989(1.452-33.645)\end{array}$ & $\begin{array}{c}- \\
0.125 \\
0.015\end{array}$ & $\begin{array}{c}- \\
2.893(0.634-\mid 3.191) \\
5.488(1.081-27.855)\end{array}$ & $\begin{array}{c}- \\
0.170 \\
0.040\end{array}$ \\
\hline FIGO stage & $\begin{array}{l}\left.|-|\right|^{R} \\
\mid I I-I V\end{array}$ & $3.335(1.212-9.177)$ & 0.020 & $2.039(0.707-5.877)$ & 0.187 \\
\hline
\end{tabular}

Note: $\mathrm{P}<0.05$ suggests significantly different.

Abbreviations: $\mathrm{MCV}$, mean corpuscular volume; $\mathrm{MCH}$, mean corpuscular hemoglobin; RDW-CV, the coefficient of variation of red blood cell distribution; $\mathrm{CA} / 25$, cancer antigen 125; CA199, cancer antigen 199; CAI53, cancer antigen 153; FIGO, International Federation of Gynecology and Obstetrics; R, references group. 

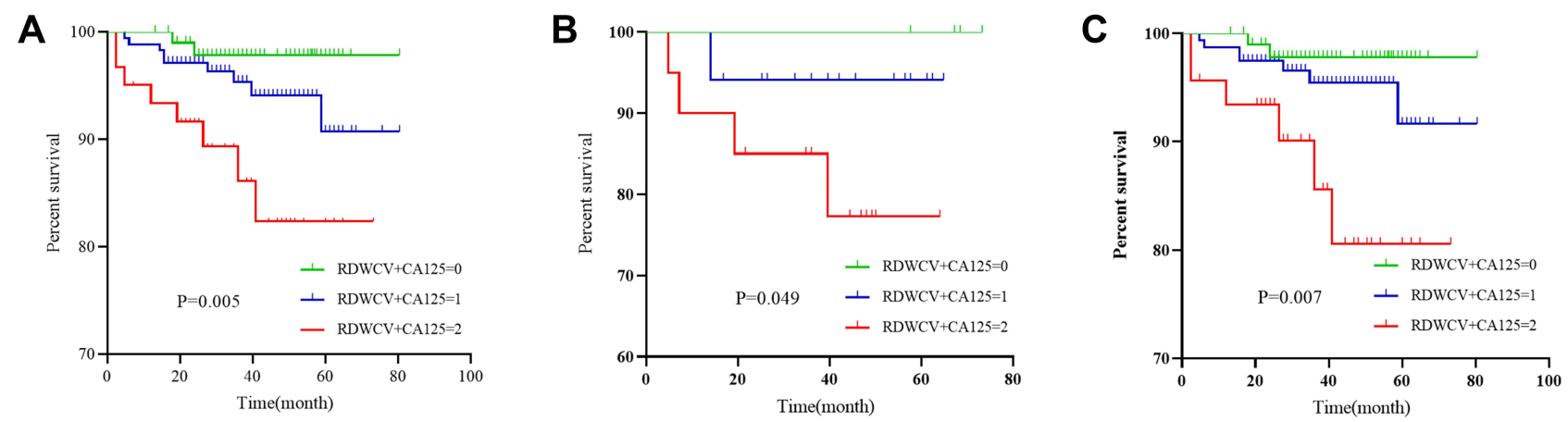

Figure 2 (A) Kaplan-Meier curve for OS of EC patients stratified by RDW-CV+CAI25. (B) Association of RDW-CV+CAI25 with the OS of EC patients with FIGO stage III-IV. (C) Association of RDW-CV+CAI 25 with the OS of EC patients with FIGO stage I-II. P<0.05 suggests significantly different.

However, there our study had some limitations. First, because this was a single-institution, retrospective study, we were unable to eliminate inherent and potential biases; therefore, a large-scale, multi-center, forward-looking study is needed to validate our conclusions. Second, we did not study the molecular mechanism, which requires further verification. In addition, recently, TCGA molecular analysis has been proved to have a good application value in the prognostic evaluation of EC. ${ }^{21-23}$ However, the value of RDW$\mathrm{CV}+\mathrm{CA} 125$ in different molecular classifications of EC was not evaluated in present study, and we need to further it in our future study. Nonetheless, this study was the first to determine the clinical value of RDW-CV and CA125 in EC and their combined use for the prognosis of EC.

In conclusion, RDW-CV+CA125 is a significant independent prognostic factor for OS in EC. Moreover, RDW-CV and CA125 are economical, rapid, simple and noninvasive method for routine blood detection. It can be used as an auxiliary evaluation indicator to help early detection of high-risk population and early treatment to improve the prognosis of EC patients. Thus, the RDW$\mathrm{CV}+\mathrm{CA} 125$ score is a promising prognostic marker for the clinical decision-making process regarding EC outcomes.

\section{Ethical Approval}

This study was approved by the ethical committee of the Department of Gynecology of Fujian Provincial Maternity and Children's Hospital. The study complied with the Declaration of Helsinki.

\section{Informed Consent}

This study was approved by the ethical committee of the Department of Gynecology of Fujian Provincial Maternity and Children's Hospital.

\section{Funding}

There is no funding to report.

\section{Disclosure}

The authors declare that they have no conflict of interest.

\section{References}

1. Ferlay J, Colombet M, Soerjomataram I, et al. Estimating the global cancer incidence and mortality in 2018: GLOBOCAN sources and methods. Int $J$ Cancer. 2019;144(8):1941-1953. doi:10.1002/ijc.31 937

2. Lee Y, Lheureux S, Oza A. Treatment strategies for endometrial cancer: current practice and perspective. Curr Opin Obstet Gynecol. 2017;29(1):47-58. doi:10.1097/GCO.0000000000000338

3. Reijnen C, Visser N, Kasius J, et al. Improved preoperative risk stratification with CA-125 in low-grade endometrial cancer: a multicenter prospective cohort study. J Gynecol Oncol. 2019;30 (5):e70. doi:10.3802/jgo.2019.30.e70

4. AlHilli M, Mariani A, Bakkum-Gamez J, et al. Risk-scoring models for individualized prediction of overall survival in low-grade and high-grade endometrial cancer. Gynecol Oncol. 2014;133(3):4 85-493. doi:10.1016/j.ygyno.2014.03.567

5. Park S, Lee S, Kim M, Kang Y, Moon H, Rhim C. Clinical characteristics of genital chlamydia infection in pelvic inflammatory disease. BMC Womens Health. 2017;17(1):5. doi:10.1186/s12905016-0356-9

6. Kokot I, Piwowar A, Jędryka M, Sołkiewicz K, Kratz E. Diagnostic significance of selected serum inflammatory markers in women with advanced endometriosis. Int J Mol Sci. 2021;22(5):2295. doi:10. 3390/ijms22052295

7. Qin C, Gao Y, Li J, Huang C, He S. Predictive effects of preoperative serum CA125 and AFP levels on post-hepatectomy survival in patients with hepatitis B-related hepatocellular carcinoma. Oncol Lett. 2021;21(6):487. doi:10.3892/ol.2021.12748

8. Feng G, Li H, Li Q, Fu Y, Huang R. Red blood cell distribution width and ischaemic stroke. Stroke Vasc Neurol. 2017;2(3):172-175. doi:10.1136/svn-2017-000071

9. He Y, Liu C, Zeng Z, Ye W, Lin J, Ou Q. Red blood cell distribution width: a potential laboratory parameter for monitoring inflammation in rheumatoid arthritis. Clin Rheumatol. 2018;37(1):161-167. doi:10. 1007/s10067-017-3871-7

10. Matsubara S, Mabuchi S, Takeda Y, Kawahara N, Kobayashi H, Robinson J. Prognostic value of pre-treatment systemic immune-inflammation index in patients with endometrial cancer. PLoS One. 2021;16(5):e0248871. doi:10.1371/journal.pone.0248871 
11. Yao D, Wang Z, Cai H, Li Y, Li B. Relationship between red cell distribution width and prognosis in patients with breast cancer after operation: a retrospective cohort study. Biosci Rep. 2019;39(7). doi:10.1042/BSR20190740

12. Qin Y, Wu Y, Xian X, et al. Single and combined use of red cell distribution width, mean platelet volume, and cancer antigen 125 for differential diagnosis of ovarian cancer and benign ovarian tumors. J Ovarian Res. 2018;11(1):10. doi:10.1186/s13048-0180382-3

13. Shota S, Saito H, Kono Y, et al. Prognostic significance of pre- and post-operative red-cell distribution width in patients with gastric cancer. J Gastrointest Surg. 2020;24(5):1010-1017. doi:10.1007/ s11605-019-04392-w

14. Tong Y, Xie X, Mao X, Lei H, Chen Y, Sun P. Low red blood cell count as an early indicator for myometrial invasion in women with endometrioid endometrial carcinoma with metabolic syndrome. Cancer Manag Res. 2020;12:10849-10859. doi:10.2147/CMAR.S271078

15. de Gonzalo-calvo D, de Luxán-delgado B, Rodríguez-González S, et al. Interleukin 6, soluble tumor necrosis factor receptor I and red blood cell distribution width as biological markers of functional dependence in an elderly population: a translational approach. Cytokine. 2012;58(2):193-198. doi:10.1016/j. cyto.2012.01.005

16. Cehreli R, Yavuzsen T, Ates H, Akman T, Ellidokuz H, Oztop I. Can inflammatory and nutritional serum markers predict chemotherapy outcomes and survival in advanced stage nonsmall cell lung cancer patients? Biomed Res Int. 2019;2019:1648072. doi:10.1155/2019/1648072
17. Wise E, Hocking $\mathrm{K}$, Weltz A, et al. Red cell distribution width is a novel biomarker that predicts excess body-mass index loss 1 year after laparoscopic Roux-en-Y gastric bypass. Surg Endosc. 2016;30 (10):4607-4612. doi:10.1007/s00464-016-4798-9

18. Zhang H, Liang K, Ke L, Tang S. Clinical application of red cell distribution width, mean platelet volume, and cancer antigen 125 detection in endometrial cancer. J Clin Lab Anal. 2020;34(8): e23309.

19. Kumar NU, Sridhar M, Srilatha K, Habebullah S. CA 125 is a better marker to differentiate endometrial cancer and abnormal uterine bleeding. Afr Health Sci. 2018;18(4):972-978. doi:10.4314/ahs. v18i4.17

20. Lin H, Ni L. Diagnostic utility of LDH, CA125 and CYFRA21-1 in tuberculosis pleural effusion. Med Clin. 2021. doi:10.1016/j. medcli.2020.10.017

21. Santoro A, Angelico G, Travaglino A, et al. New pathological and clinical insights in endometrial cancer in view of the updated ESGO/ ESTRO/ESP guidelines. Cancers. 2021;13(11):2623. doi:10.3390/ cancers13112623

22. Raffone A, Travaglino A, Raimondo D, et al. Prognostic value of myometrial invasion and TCGA groups of endometrial carcinoma. Gynecol Oncol. 2021;162(2):401-406. doi:10.1016/j.ygyno.2021.05. 029

23. Raffone A, Travaglino A, Gabrielli O, et al. Clinical features of ProMisE groups identify different phenotypes of patients with endometrial cancer. Arch Gynecol Obstet. 2021;303(6):1393-1400. doi:10.1007/s00404-021-06028-4
International Journal of General Medicine

\section{Publish your work in this journal}

The International Journal of General Medicine is an international, peer-reviewed open-access journal that focuses on general and internal medicine, pathogenesis, epidemiology, diagnosis, monitoring and treatment protocols. The journal is characterized by the rapid reporting of reviews, original research and clinical studies

\section{Dovepress}

across all disease areas. The manuscript management system is completely online and includes a very quick and fair peer-review system, which is all easy to use. Visit http://www.dovepress.com testimonials.php to read real quotes from published authors. 